\title{
The Roles of the LIM Domain Proteins in Drosophila Cardiac and Hematopoietic Morphogenesis
}

\author{
Meihua She ${ }^{1 \dagger}$, Min Tang ${ }^{1 \dagger}$, Tingting Jiang ${ }^{2 t}$ and Qun Zeng ${ }^{1 *}$ \\ ${ }^{1}$ Department of Biochemistry and Molecular Biology, College of Hengyang Medical, University of South China, Hengyang, \\ China, ${ }^{2}$ Affiliated Nanhua Hospital, University of South China, Hengyang, China
}

Drosophila melanogaster has been used as a model organism for study on development and pathophysiology of the heart. LIM domain proteins act as adaptors or scaffolds to promote the assembly of multimeric protein complexes. We found a total of 75 proteins encoded by 36 genes have LIM domain in Drosophila melanogaster by the tools of SMART, FLY-FISH, and FlyExpress, and around $41.7 \%$ proteins with LIM domain locate in lymph glands, muscles system, and circulatory system. Furthermore, we summarized functions of different LIM domain proteins in the development and physiology of fly heart and hematopoietic systems. It would be attractive to determine whether it exists a probable "LIM code" for the cycle of different cell fates in cardiac and hematopoietic tissues. Next, we aspired to propose a new research direction that the LIM domain proteins may play an important role in fly cardiac and hematopoietic morphogenesis.

Reviewed by:

Maria Spletter,

Ludwig Maximilian University of

Munich, Germany

Henry J. Duff,

University of Calgary, Canada

Lina Worpenberg,

University of Lausanne, Switzerland

*Correspondence:

Qun Zeng

zengqun2017@163.com

†These authors have contributed equally to this work

Specialty section:

This article was submitted to Cardiovascular Genetics and Systems

Medicine,

a section of the journal

Frontiers in Cardiovascular Medicine

Received: 21 October 2020

Accepted: 04 January 2021

Published: 11 February 2021

Citation:

She $M$, Tang $M$, Jiang $T$ and Zeng $Q$ (2021) The Roles of the LIM Domain

Proteins in Drosophila Cardiac and Hematopoietic Morphogenesis.

Front. Cardiovasc. Med. 8:616851.

doi: 10.3389/fcvm.2021.616851

\section{INTRODUCTION}

The Drosophila melanogaster has emerged as a powerful model for studying heart development and cardiac diseases. Using the tools of both classical and molecular genetics, the study of the phylogeny of fly heart has been influential in the denomination of the primary signaling events of cardiac area formation, cardiomyocyte specification, and the formation of the functioning heart tube (1). Several studies that are underway may take advantage of the fruit fly as an in vivo model for exploring genes involved in cardiac formation. Numerous mutations in conserved hereditary pathways have been found, including those commanding development and physiology of the heart (2). The function and expression pattern of the homeobox transcription factor Tinman in Drosophila and its vertebrate homologous NKX2.5 are remarkably similar in the embryology of heart tube morphogenesis and heart function, and this strikingly provides the compelling evidence that heart development is controlled by conserved homologous pathways in both invertebrates and vertebrates (2-6). After that, many transcription factors, and signal pathways involved in the cardiac specification has been elucidated. Pnr is essential in the mesoderm for initiation of cardiac-specific expression of tinman and for specification of the heart primordium (7). Wnt signaling activated by $\mathrm{Wg}$, together with $\mathrm{Dpp}$, is required for specification of cardiac progenitor cells in early heart development (8). These basic determinants of cardiogenesis in the fly play elementary roles in both the immature cardiac specification and heart function in the adult fly and human (4).

Heart progenitors of flies are bilaterally symmetrical, distributed in most of dorsal regions of the mesoderm in Drosophila (9). During cardiogenesis, these progenitors migrate to the dorsal midline and form the heart tube, a stretchable linear tube, which is consisted of two different types of cells, 
ie. contractile myocardial cells in the inner layer and noncontractile pericardial cells. Pericardial cells are the excretory cells, called pericardial nephrocytes $(10,11)$. Drosophila melanogaster also shares with vertebrates the basic regulatory mechanisms and genetic control of hematopoiesis, making it a good model for solving outstanding problems in blood development $(12,13)$. Lymph organ, the hematopoietic system in Drosophila, which produces the hemolymph cells, is derived from the cardiac progenitors, some genes that affect heart development may also be involved in regulating the differentiation of blood cells (14). Drosophila has three classes of blood cells: plasmatocytes, lamellocytes, and crystal cells. The development of crystal cells, a non-phagocytic cell, is similar to the vertebrate erythroid development in the early phase $(15,16)$. Despite its simple structure, the fly heart has recently emerged as an admirable model system for dissecting complicated pathways that determine the fate of cardiac cells and studying the physiological functions of the adult heart (17).

LIM is a small protein-protein synergism domain containing two zinc fingers (18). LIM domains are identified in a dissimilar group of proteins with a wide variety of biological roles, including gene expression regulation, cell fate determination, cytoskeleton organization, tumor formation, and embryonic development (19). LIM domains function as adaptors or scaffolds to support the assembly of multimeric protein complexes via interacting with alteration protein partners. LIM domains generally have about 50-60 amino acids and are characterized by two highly conserved zinc finger motifs. These zinc fingers contain eight conserved residues, generally with cysteines and histidines coordinately binding to two zinc atoms. The consensus sequence of LIM domain has been defined as C-X(2)-C-X(16, 23)-H$\mathrm{X}(2)-[\mathrm{CH}]-\mathrm{X}(2)-\mathrm{C}-\mathrm{X}(2)-\mathrm{C}-\mathrm{X}(16,21)-\mathrm{C}-\mathrm{X}(2,3)-[\mathrm{CHD}]$ (where $\mathrm{X}$ denotes any amino acid) (18-20).

In this review, Firstly, we found a total of 75 proteins encoded by 36 genes have LIM domain in Drosophila melanogaster by the tools of SMART, and most proteins with LIM domain locate in lymph glands, muscles system, and circulatory system according to FLY-FISH, and FlyExpress. Secondly, we summarized functions of four LIM domain proteins in the development and physiology of fly heart and hematopoietic systems. Lastly, we aspired to propose a new research direction that other LIM domain proteins may play an important role in fly cardiac and hematopoietic morphogenesis. Based on the expression parrerns, and biological roles of the LIM domain, we speculated that it has an important function involved in Drosophila heart development, heart function, and/or hematopoietic morphogenesis. Therefore, this review focus on the roles of LIM domain proteins in Drosophila cardiac and hematopoietic morphogenesis.

\section{Bioinformatics Analysis of Drosophila LIM Domain Proteins}

SMART (Simple Modular Architecture Research Tool) is a web resource for the identification and annotation of protein domains and the analysis of protein domain architectures (http://smart. embl.de) $(21,22)$. We used the SMART tool to identify proteins containing the LIM domain in Drosophila melanogaster. It is expected that a total of 36 genes encoding 75 proteins with the LIM domain in Drosophila melanogaster (Table 1). According to the characteristics of protein domains, LIM-domain proteins were classified into three types, LIM proteins, which include the LIM domain and other protein structures; LIM-only types with only the LIM domain; and LIM-homeodomain (LIMHD) types, which include the LIM domain and homeodomain domain. These genes participate in the biological functions of various organs and tissues such as muscle, heart, lymph gland, nervous system, eye development, and leg morphogenesis, and so on (Table 1). Then we evaluated the expression regions of these genes in fruit flies by two databases. One is FlyFISH (http://fly-fish.ccbr.utoronto.ca/), a database documents the expression and localization patterns of Drosophila mRNAs at the cellular and subcellular level during early embryogenesis and third instar larval tissues (Figures 2G-M) $(23,24)$. The other is FlyExpress (http://www.flyexpress.net/), a freely approachable online knowledgebase offering users an occasion to investigate and analyze expression patterns of developmental genes in Drosophila embryogenesis (Figures 2A-F) (25-27). According to the FLY-FISH or FlyExpress databases, there are 15 LIM genes expressed in mesoderm including muscles, heart, or lymph glands (Figure 1 Columm A). It indicates that 11 LIM genes are expressed in other non-Columm A tissues (Figure 1 Columm B). The expression patterns of the remaining 10 LIM genes are unclear (Figure 1 Columm C). From these two databases, we perceived that some of the 36 genes were expressed in the heart (Table 1 and Figures 2A-J), lymph gland (Table 1 and Figures $2 \mathbf{K}-\mathbf{M}$ ), muscle, CNS, and amnioserosa (Table 1), and so on. In general, around $41.7 \%$ Drosophila LIM domain genes were enriched expressed in mesoderm including muscles, heart, or lymph glands (Figure 1 Columm A), and this signified that these genes might have a important role in heart, and/or hematopoietic development. In the accompanying sections, we introduced some specific LIM domain proteins, tailup, mlp84B, radish, and Beadex, which have been confirmed by scientists, play roles in cardiac, and/or hematopoietic morphogenesis. However other LIM domain genes, which enriched expressed in heart, lymph glands, or other tissues, whether it is related to heart development, heart function, or hematopoietic morphogenesis remains to be further studied. In a word, we provide a new idea for the future research.

\section{LIM Homeodomain Transcription Factor tailup Is Required for Normal Heart and Hematopoietic Organ Formation in Drosophila}

Fly LIM homeodomain transcription factor tailup (Tup), a homologous gene of Islet 1 in vertebrate, belongs to LIM-HD type, expresses in all cardiac cells (CC) and pericardial cells (PC) of the heart tube as well as lymph glands hematopoietic organs (28-30), which is not in line with the data in Table 1, we think it didn't be detected, or the probes did not work in the two databases (FLY-FISH, and FlyExpress). In vertebrates, the homologous gene Islet1 is also a cardiac progenitor marker (31), and many of the heart formation processes are regulated by the transcription 
TABLE 1 | List of the various transcripts and proteins with LIM domain in Drosophila melanogaster.

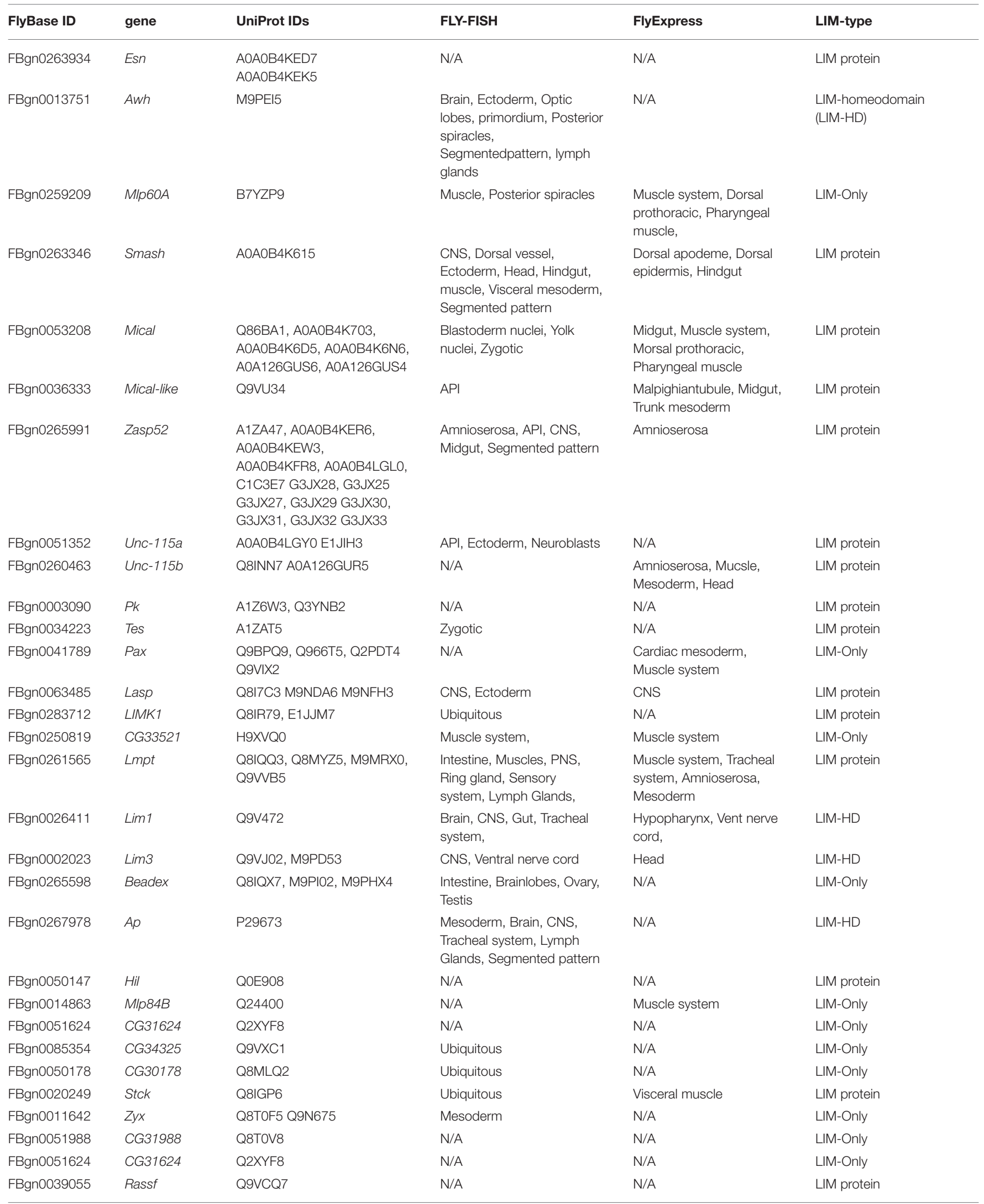


TABLE 1 | Continued

\begin{tabular}{|c|c|c|c|c|c|}
\hline FlyBase ID & gene & UniProt IDs & FLY-FISH & FlyExpress & LIM-type \\
\hline FBgn0003896 & Tup & Q9VJ37 & $\begin{array}{l}\text { Amnioserosa, API, Foregut, } \\
\text { Head, Tracheal system, } \\
\text { Muscles, Dorsal vessel }\end{array}$ & $\begin{array}{l}\text { Amnioserosa, Leading } \\
\text { edge cell, CNS, Dorsal } \\
\text { epidermis, Embryonic } \\
\text { brain, sophagus, Foregut, } \\
\text { Stomatogastric }\end{array}$ & LIM-HD \\
\hline FBgn0032196 & CG5708 & Q9VL21 & $\mathrm{N} / \mathrm{A}$ & NA & LIM-Only \\
\hline FBgn0036274 & CG4328 & Q9VTW3 & Ventral nerve, cord,CNS & NA & LIM-HD \\
\hline FBgn0052105 & $L m \times 1 a$ & Q9VTW5 & Ventral nerve cord,CNS & $\begin{array}{l}\text { CNS, Trunk mesoderm, } \\
\text { Longitudinal visceral } \\
\text { mesoderm }\end{array}$ & LIM-HD \\
\hline FBgn0030530 & $J u b$ & Q9VY77 & $\mathrm{N} / \mathrm{A}$ & $\mathrm{N} / \mathrm{A}$ & LIM-Only \\
\hline FBgn0265597 & Rad & $\begin{array}{l}\text { X2JBl1 } \\
\text { X2JDH0 }\end{array}$ & N/A & $\mathrm{N} / \mathrm{A}$ & LIM protein \\
\hline
\end{tabular}

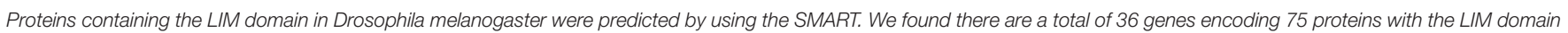
in Drosophila melanogaster. These genes play roles in a variety of biological functions, including muscle, heart, lymph gland, nervous system, eye development, and leg morphogenesis, and so on. According to the characteristics of protein domains, LIM proteins were classified into three types, LIM protein types, LIM-only types, LIM-homeodomain (LIM-HD) types.

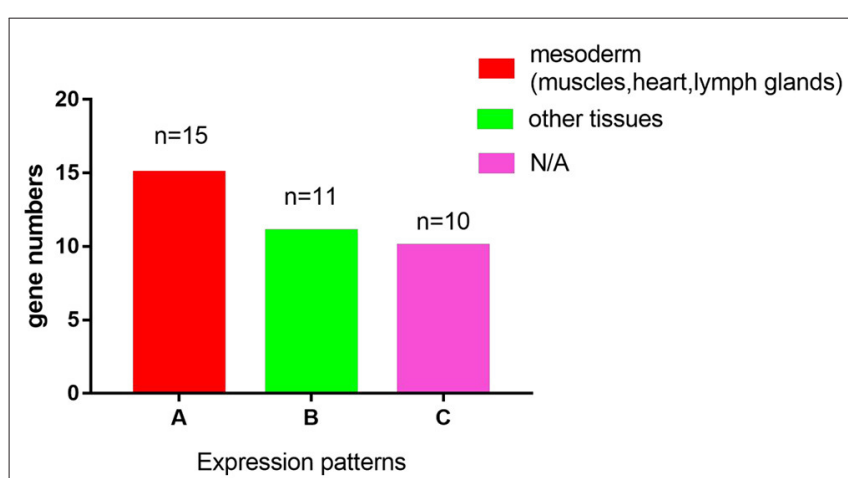

FIGURE 1 | A graphic overview of the expression patterns of the 36 LIM genes in Drosophila melanogaster. According to the FLY-FISH or FlyExpress databases, there are $15 \mathrm{LIM}$ genes expressed in mesoderm including muscles, heart, or lymph glands (Columm A). It indicates that 11 LIM genes are expressed in other non- Columm A tissues (Columm B). The expression patterns of the remaining 10 LIM genes are unclear (Columm C).

factor Islet1 (32). It is reported that, in Tup mutant fly embryos, heart tubes showed misaligned cardio-blasts and lost most lymph glands and pericardial cells (33). This indicated Tup participated in fly heart development. While irregular morphology of the heart with hypertrophied lymph glands has been observed when Tup was overexpressed in fly mesoderm (33). This indicated Tup also participated in fly hematopoietic organ formation. Tup is an entrant in the regulatory network controlling dorsal vessel morphogenesis and hematopoietic organ formation. Tup can be considered to be a main upstream regulator of genetic and cellular events controlling lymph gland formation (33). For example, it is reported that Tup was an upstream regulator of Hand in these developmental processes. Tup can recognize and bind to two DNA response sequences in the enhancer of the Hand, which has been proven to be essential for the lymph gland cells, pericardial cells, and cardiac cells (34). Also, Tup is a regulator of $\operatorname{srp}$ and $o d d$, which play an essential role in hematopoietic progenitor cell specification $(33,35)$. Drosophila LIM-HD domain protein Tup was specifically expressed in heart lymph gland tissue and had a unique role in heart development. LIM-domain factors affiliated to cooperate with the adaptor snippet Chip/Ldb1 to form higher-order protein complexes to determine gene expression (36). In a word, Tup interacted with Chip/Ldb1 to regulate some ingenious factors, such as Even-skipped (Eve), Hand, and Odd, involved in cardiac cell specification and differentiation (37-40). As we known, the GATA factors $p n r$ and $s r p$ directly activate Hand in cardioblasts, pericardial nephrocytes and hematopoietic progenitors, Hand is initially expressed weak and segmental in cardiac cells, but soon becomes strong in most cardioblasts and pericardial cells from embryo in stage 13 to adult heart. The pattern expression of hand is controlled by a 513bp enhancer between exons 3 and 4 of the Hand gene, which contained DNA sequence with Tinman- and GATA-binding sites (41).

Due to the expression patterns and functions of Tup in fly heart, as well as some other LIM-HD type genes listed in the Table 1, which expressed in the mesoderm at early stage, it is implied that LIM-HD domain proteins might regulate the differentiation and specialization of cardiac progenitor cells in early cardiac development, and interacte with Chip/Ldb1 to determine normal heart and hematopoietic organ formation in Drosophila (36). Also, some genes involved in heart development could be the targets of LIM-HD domain proteins.

\section{MIp84B, a Muscle LIM Protein, Is Essential for Cardiac Function in Drosophila}

$M l p 84 B$, a muscle LIM protein (MLP), belongs to the LIM-only type, locates at the Z-disc of sarcomeres $(42,43)$. The human MLP gene has been represented to be a key player in the stretchsensing response, and its mutant is associated with hypertrophic and dilated cardiomyopathy (44). In mice, the LIM domain protein CRP2, a Drosophila Mlp84B homolog, expressed in the 

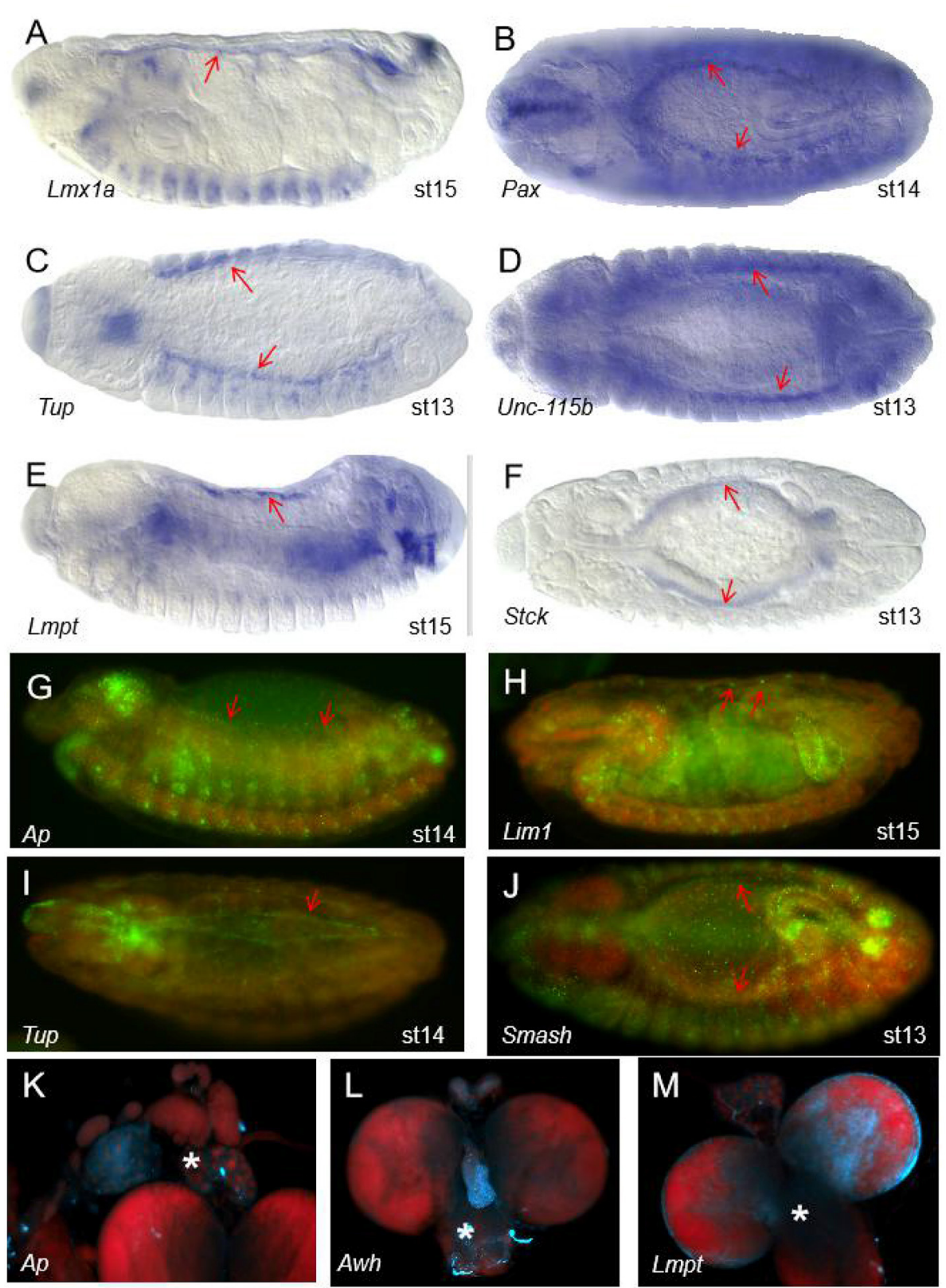

FIGURE 2 | The expression patterns of some of the 36 LIM genes in Drosophila melanogaster. (A-F) Expression patterns of some genes with the LIM domain in wild type (WT) Drosophila embryos from the FlyExpress (ISH) database. The red arrows represent the heart region of the fruit fly embryo. Embryos are at stage 13-15 of development. Lmx1a (A), Pax (B), Tup (C), Unc-115b (D), Lmpt (E), and Stck (F) are expressed in Drosophila heart tissue. (G-J) Expression patterns of some genes with the LIM domain in Drosophila embryos in the Fly-FISH database. The red arrows represent the heart region of the fruit fly embryo. Embryos are at stage 13-15 of development. Ap (G), Lim1 (H), Tup (I), and Smash (J) are expressed in Drosophila heart tissue. Embryo colors: Red/DNA, Green/RNA. (K-M) The area of the lymph glands in the anatomy. Expression patterns of some genes with the LIM domain in the third larvae of Drosophila in the Fly-FISH database. The white asterisk represents the lymph gland region of the fruit fly larvae. Ap (K), Awh (L), and Lmpt (M) are expressed in Drosophila lymph gland. Larval colors: Red/DNA, Blue/RNA.

vascular system, especially in smooth muscle cells. CRP2 knockout mice exhibit some mild hypertrophy phenotype in their cardiac ultrastructure (45). $M l p 84 B$, co-localizes with $\alpha$-actinin in the heart from late embryonic stages to adulthood in Drosophila, and is essential for Drosophila lifespan and cardiac function $(46,47)$. The shortened lifespan was found in $M l p 84 B$ knockout flies, and with a dramatically decreased lifespan in knockdown flies by the cardiac driver tinC $\Delta 4-\mathrm{Gal} 4$, which are due to the role of Mlp84B in cardiac function. Mlp84B mutant flies appeared bradycardia and heart rhythm abnormalities without obvious organic phenotype (46). We believe that MLP proteins may play a critical role in the differentiation of cardiac muscle and 


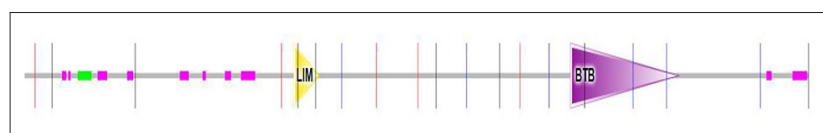

FIGURE 3 | Domains within Drosophila melanogaster protein Radish. Yellow triangle box represents the LIM domain region, the LIM domain of Radish was located at positions 568 to 622 amino acids.

contribute to proper cardiac function by directing cardiac muscle structure development. It is known that $d M E F 2$, an invertebrate member of the Myocyte Enhancer Factors family of vertebrate myocyte, has MADS-box and MEF2 domains which assisted its dimerization and DNA binding, plays an essential role in muscle differentiation (48). Due to the six dMEF2 binding sites found in the $M l p 84 B$ locus, $d M E F 2$ is necessary for the expression of $M l p 84 B$ in all the muscle tissues including skeletal, cardiac, and smooth muscles (48). Collectively, it suggested that Mlps may be targets of dMEF2 proteins that contribute to cardiomyocyte differentiation and function. We hypothesized that LIM domain genes expressed in muscle tissue are likely regulated by MEF2, which determines the differentiation of the three types of muscle cells. We predicted LIM genes expressed in heart muscle tissue, such as Mlp60A, Smash, Lmpt, etc (see Table 1), might be essential for the differentiation of cardiomyocyte.

\section{The Drosophila radish Gene Is Required for Cardiac Pacemaker}

The Drosophila radish (Rad) gene is required for cardiac function (49). It is known that Rad, encoding a protein with a POZ domain and a Rap GTPase activating protein domain, involved in regulating anesthesia-resistant memory and heart contraction function $(49,50)$. Interestingly, we found that $\mathrm{Rad}$ is also a member of the LIM protein family, based on the predictions of SMART tool (Table 1). We also analyzed the domains within Drosophila melanogaster protein Radish, the LIM domain was located at positions 568 to 622 amino acids (Figure 3). Conveniently, there is no expression data presented for Rad in Table 1, further studies are needed to confirm whether it is expressed in heart tissue. The exceptional Rad mutants, which effected memory and retention, substantially lessened heart rate and rhythms. The heart rate of Rad mutants was significantly lower than that of wild mutants at different temperatures (49). Besides, these mutants were insensitive to both serotonin and norepinephrine. In a word, Rad mutations were closely associated with bradycardia in fly. Its effect on heart function is likely mediated via neurotransmitter release (49). While the molecular mechanism by which the Rad regulates heart function remained ambiguous, Rad likely effects the heart modulatory pathway (49). We knew that muscle LIM protein Mlp84B, mainly located at the Z-line boundary, maintained the structural integrity of muscles by interacting with $\alpha$-actinin, and D-titin was essential for cardiac function $(47,51)$. We hypothesized the LIM proteins, such as Rad, if it is expressed in the heart muscle tissue, it maybe also interacted with the modular protein present in muscles to regulate the structure of the heart muscles and thus affected the function of the heart. So LIM proteins not only regulate cardiac development but also affect cardiac function.

\section{Beadex, a Drosophila Homolog of LIM Domain Only 2 (LMO2), Functions in Hematopoiesis}

The lymph organ, which produces the hemolymph cells, is derived from the cardiac progenitors, some of the genes that affect heart development also involved in hematopoiesis. Tup, a regulator of $\operatorname{srp}$ and odd expression in lymph gland cells and pericardial cells $(33,35)$, is a typical example. So we think that in addition to affecting heart function and heart development, LIM proteins also regulate blood cell formation in Drosophila. There are three classes of blood cells in Drosophila: plasmatocytes, lamellocytes, and crystal cells (17, 52). The development of crystal cell, a non-phagocytic cell, is similar to that of vertebrate erythroid in the early phase (53). In Table 1, we found that Beadex, a Drosophila homolog of LIM domain only 2 (LMO2), is expressed in hemocytes, and was reported to effect the numbers of crystal cells after their specification (54). Morever, in vertebrate, $L M O 2$ is necessary for the emergence of hematopoietic stem cells during ontogeny and angiogenesis by modeling a complex with the LIM domain-binding protein 1, and binding to the E-box and GATA transcription factors $(55,56)$. In fly, overexpression of Beadex in crystal cell lineage leading to a dramatic increase in the number of crystal cells. Meanwhile, a knockdown of Beadex resulted in a significant decrease in the number of crystal cells (3). This indicated Beadex is involved in the proliferation of blood cells in fly. Drosophila GATA factor pannier (Pnr), a known interacting colleague of Beadex. Gain of function (GOF) of both pnr and Beadex in fly, the crystal cell counts were consistent with that of pnr GOF alone, and GOF of Beadex and loss of function (LOF) of pnr in fly, the crystal cell counts were similar to that of pnr LOF alone, therefore, the misexpression of pnr masked the effect of Beadex on the number of crystal cells. So, $p n r$ has genetic interactions with Beadex during the development of crystal cell $(3,57,58)$. Pnr, as a downstream molecular, can bind to cofactor U-shaped (Ush) to inhibite crystal cell development $(3,59)$. Thus, pnr is also supposed to interfere in the development of crystal cells. That is to denote, LIM proteins might contribute to hematopoietic development through the GATA transcription factor. According to the Fly-FISH database, we also found that some LIM genes expressed in the lymph glands of Drosophila (Table $\mathbf{1}$ and Figures $2 \mathrm{~K}-\mathbf{M})$. Combined with the study on the role of Beadex in the hematopoietic system in Drosophila, we speculate that LIM proteins may also have a role in the hematopoietic system of Drosophila.

\section{PERSPECTIVES}

This review summarized the role and molecular mechanisms of LIM transcription factors of Drosophila during cardiovascular development. The Drosophila melanogaster has emerged as a powerful model for studying heart development and cardiac diseases, as well as an excellent model for human cardiac 
development and diseases $(17,52)$. LIM domains are identified in diverse groups of proteins with a wide variety of biological functions, including gene expression regulation, cell fate determination, cytoskeleton organization, tumor formation, and heart development (18-20). Just as LIM related factors arise to be conserved in the arrangement of cardiac development and physiology in the animal kingdom from fly to human.

Through analysis of the bioinformatics database of Drosophila LIM domain transcription factors, a total of 36 genes encoding 75 proteins with the LIM domain in Drosophila melanogaster were found. We divided LIM proteins into three categories, LIM protein type, LIM-only type, and LIM-HD type, according to the characteristics of protein domains. LIM domain proteins can be either transcription factors or structural proteins to regulate cardiac and hematopoietic morphogenesis. As a transcription factor, such as tup, can recognize and bind to two DNA response sequences in the enhancer of the Hand, and act as a regulator of $\operatorname{srp}$ and odd to determines the differentiation of lymph gland cells, cardiac cells, and pericardial cells. As a structural protein, such as Mlp84B, is the cytoskeletal protein, directly affects muscle structure and function. From Fly-FISH and FlyExpress (23-27), we knew that some of LIM domain mRNAs were located in lymph glands, muscles system, and circulatory system, which inferred that LIM domain genes might have roles in cardiac and hematopoietic morphogenesis. It would be attractive to dissect whether it exists a probable LIM code for the cycle of different cell fates in cardiac and hematopoietic tissues, and this hypothesis needs to be better supported in future studies. In this review, we aspired to proposed a research direction that the LIM domain genes may

\section{REFERENCES}

1. Frasch M. Genome-wide approaches to Drosophila heart development. J Cardiovasc Dev Dis. (2016) 3:20. doi: 10.3390/jcdd3020020

2. Bodmer R, Venkatesh T. Heart development in Drosophila and vertebrates: conservation of molecular mechanisms. Dev Genet. (1998) 22:181-6. doi: 10. 1002/(SICI)1520-6408(1998)22:3 <181::AID-DVG1>3.0.CO;2-2

3. Chatterjee A, Aavula K, Nongthomba U. Beadex, a homologue of the vertebrate LIM domain only protein, is a novel regulator of crystal cell development in Drosophila melanogaster. J Genet. (2019) 98:107. doi: 10.1007/s12041-019-1154-6

4. Berrak U, Kuchuan C, Hugo JB. Drosophila tools and assays for the study of human diseases. Dis Model Mech. (2016) 9:235-44. doi: 10.1242/dmm.023762

5. Bodmer R. The gene tinman is required for specification of the heart and visceral muscles in Drosophila. Development. (1993) 118:719-29.

6. Cannon L, Bodmer R. Genetic manipulation of cardiac ageing. J Physiol. (2016) 594:2075-83. doi: 10.1113/JP270563

7. Klinedinst SL, Bodmer R. Gata factor Pannier is required to establish competence for heart progenitor formation. Development. (2003) 130:3027-38. doi: 10.1242/dev.00517

8. Lockwood WK, Bodmer R. The patterns of wingless, decapentaplegic, and tinman position the Drosophila heart. Mech Dev. (2002) 114:13-26. doi: 10.1016/S0925-4773(02)00044-8

9. Rotstein B, Paululat A. On the morphology of the Drosophila heart disease. $J$ Cardiovasc Dev Dis. (2016) 3:15. doi: 10.3390/jcdd3020015

10. Crossley A. The ultrastructure and function of pericardial cells and other nephrocytes in an insect: calliphora erythrocephala. Tissue Cell. (1972) 4:52960. doi: 10.1016/S0040-8166(72)80029-6 play important roles in cardiac development and hematopoiesis. Cardiomyocytes are a type of muscle cells, and $\mathrm{dMef} 2$ regulates the differentiation of cardiomyocytes, therefore, we hypothesized that one possible mechanism is that LIM domain genes can act as the targets of dMef2 in heart tissue, regulate the differentiation of cardiomyocytes, interact with Chip/Ldb1 to determine normal heart and hematopoietic organ formation in Drosophila, and participate in hematopoietic development through GATA transcription factor.

\section{AUTHOR CONTRIBUTIONS}

MS and QZ wrote and edited the manuscript together. MT and TJ generated the figures and table together. All authors contributed to the article and approved the submitted version.

\section{FUNDING}

This work was supported by the Natural Science Foundation of Hunan Province (No.2020JJ5479), Hunan Provincial Department of Education Project (No.19C1604), Doctoral Research Foundation of University of South China (No.190XQD022), and University Student Innovation Project of University of South China (No.X2019153).

\section{ACKNOWLEDGMENTS}

We are very grateful to Kumar S. for permitting us to use the images in FlyExpress and Henry Krause for permitting us to use the images in Fly-FISH.

11. Villanueva J, Livelo C, Trujillo A, Chandran S, Woodworth B, Andrade L, et al. Time-restricted feeding restores muscle function in Drosophila models of obesity and circadian-rhythm disruption. Nat Commun. (2019) 10:2700. doi: 10.1038/s41467-019-10563-9

12. Ramond E, Meister M, Lemaitre B. From embryo to adult: hematopoiesis along the Drosophila life cycle. Dev Cell. (2015) 33:367-8. doi: 10.1016/j.devcel.2015.05.002

13. Koranteng F, Cha N, Shin M, Shim J. The role of lozenge in Drosophila hematopoiesis. Mol Cells. (2020) 43:114-20. doi: 10.14348/molcells.2019.0249

14. Mandal L, Banerjee U, Hartenstein V. Evidence for a fruit fly hemangioblast and similarities between lymph-gland hematopoiesis in fruit fly and mammal aorta-gonadal-mesonephros mesoderm. Nature genetics. (2004) 36:1019-23. doi: $10.1038 /$ ng1404

15. Banerjee U, Girard JR, Goins LM, Spratford CM. Drosophila as a genetic model for hematopoiesis. Genetics. (2019) 211:367-417. doi: 10.1534/genetics.118.300223

16. Evans C, Hartenstein V, Banerjee U. Thicker than blood: conserved mechanisms in Drosophila and vertebrate hematopoiesis. Dev Cell. (2003) 5:673-90. doi: 10.1016/S1534-5807(03)00 335-6

17. Crozatier M, Vincent A. Drosophila: a model for studying genetic and molecular aspects of haematopoiesis and associated leukaemias. Dis Model Mech. (2011) 4:439-45. doi: 10.1242/dmm.007351

18. Freyd G, Kim S, Horvitz H. Novel cysteine-rich motif and homeodomain in the product of the Caenorhabditis elegans cell lineage gene lin-11. Nature. (1990) 344:876-9. doi: 10.1038/344876a0

19. Karlsson O, Thor S, Norberg T, Ohlsson H, Edlund $T$. Insulin gene enhancer binding protein Isl-1 is a member of a novel class of proteins 
containing both a homeo- and a Cys-His domain. Nature. (1990) 344:879-82. doi: $10.1038 / 344879 \mathrm{a} 0$

20. Liang Y, Bradford WH, Zhang J, Sheikh F. Four and a half LIM domain protein signaling and cardiomyopathy. Biophys Rev. (2018) 10:1073-85. doi: 10.1007/s12551-018-0434-3

21. Mende DR, Letunic I, Huerta-Cepas J, Li SS, Forslund K, Sunagawa S, et al. proGenomes: a resource for consistent functional and taxonomic annotations of prokaryotic genomes. Nucleic Acids Res. (2017) 45:D529-34. doi: 10.1093/nar/gkw989

22. Finn RD, Attwood TK, Babbitt PC, Bateman A, Bork P, Bridge AJ, et al. InterPro in 2017-beyond protein family and domain annotations. Nucleic Acids Res. (2017) 45:D190-9. doi: 10.1093/nar/gkw1107

23. Lécuyer E, Yoshida H, Parthasarathy N, Alm C, Babak T, Cerovina T, et al. Global analysis of mRNA localization reveals a prominent role in organizing cellular architecture and function. Cell. (2007) 131:174-87. doi: 10.1016/j.cell.2007.08.003

24. Wilk R, Hu J, Blotsky D, Krause H. Diverse and pervasive subcellular distributions for both coding and long noncoding RNAs. Genes Dev. (2016) 30:594-609. doi: 10.1101/gad.276931.115

25. Konikoff C, Karr T, McCutchan M, Newfeld S, Kumar S. Comparison of embryonic expression within multigene families using the FlyExpress discovery platform reveals more spatial than temporal divergence. Dev Dyn. (2012) 241:150-60. doi: 10.1002/dvdy.22749

26. Kumar S, Boccia K, McCutchan M, Ye J. Exploring spatial patterns of gene expression from fruit fly embryogenesis on the iPhone. Bioinformatics. (2012) 28:2847-8. doi: 10.1093/bioinformatics/bts518

27. Kumar S, Konikoff C, Van Emden B, Busick C, Davis K, Ji S, et al. FlyExpress: visual mining of spatiotemporal patterns for genes and publications in Drosophila embryogenesis. Bioinformatics. (2011) 27:3319-20. doi: 10.1093/bioinformatics/btr567

28. Boukhatmi H, Schaub C, Bataillé L, Reim I, Frendo J, Frasch M, et al. An Org-1-Tup transcriptional cascade reveals different types of alary muscles connecting internal organs in Drosophila. Development. (2014) 141:3761-71. doi: 10.1242/dev.111005

29. Cai C, Liang X, Shi Y, Chu P, Pfaff S, Chen J, et al. Isl1 identifies a cardiac progenitor population that proliferates prior to differentiation and contributes a majority of cells to the heart. Dev Cell. (2003) 5:877-89. doi: 10.1016/S1534-5807(03)00363-0

30. Zmojdzian M, Jagla K. Tailup plays multiple roles during cardiac outflow assembly in Drosophila. Cell Tissue Res. (2013) 354:639-45. doi: 10.1007/s00441-013-1644-4

31. Foo KS, Lehtinen ML, Leung CY, Lian X, Xu J, Keung W, et al. Human ISL1(+) ventricular progenitors self-assemble into an in vivo functional heart patch and preserve cardiac function post infarction. Mol Ther. (2018) 26:1644-59. doi: 10.1016/j.ymthe.2018.02.012

32. Hatzistergos KE, Durante MA, Valasaki K, Wanschel ACBA, William Harbour J, Hare JM. A novel cardiomyogenic role for Isl1 ${ }^{+}$neural crest cells in the inflow tract. Sci Adv. (2020) 6:eaba9950. doi: 10.1126/sciadv.aba9950

33. Tao Y, Wang J, Tokusumi T, Gajewski K, Schulz R. Requirement of the LIM homeodomain transcription factor tailup for normal heart and hematopoietic organ formation in Drosophila melanogaster. Mol Cell Biol. (2007) 27:3962-9. doi: 10.1128/MCB.00093-07

34. Zaffran S, Reim I, Qian L, Lo P, Bodmer R, Frasch M. Cardioblastintrinsic Tinman activity controls proper diversification and differentiation of myocardial cells in Drosophila. Development. (2006) 133:4073-83. doi: $10.1242 /$ dev.02586

35. Sorrentino R, Gajewski K, Schulz R. GATA factors in Drosophila heart and blood cell development. Semin Cell Dev Biol. (2005) 16:107-16. doi: 10.1016/j.semcdb.2004.10.005

36. Franz A, Shlyueva D, Brunner E, Stark A, Basler K. Probing the canonicity of the Wnt/Wingless signaling pathway. PLoS Genet. (2017) 13:e1006700. doi: 10.1371/journal.pgen.1006700

37. Bronstein R, Segal D. Modularity of CHIP/LDB transcription complexes regulates cell differentiation. Fly. (2011) 5:200-5. doi: 10.4161/fly.5.3.14854

38. Caputo L, Witzel HR, Kolovos P, Cheedipudi S, Looso M, Mylona A, et al. The Isl1/Ldb1 complex orchestrates genome-wide chromatin organization to instruct differentiation of multipotent cardiac progenitors. Cell Stem Cell. (2015) 17:287-99. doi: 10.1016/j.stem.2015.08.007
39. de Navascués J, Modolell J. The pronotum LIM-HD gene tailup is both a positive and a negative regulator of the proneural genes achaete and scute of Drosophila. Mech Dev. (2010) 127:393-406. doi: 10.1016/j.mod.2010.05.001

40. Jin H, Stojnic R, Adryan B, Ozdemir A, Stathopoulos A. Frasch M. Genomewide screens for in vivo Tinman binding sites identify cardiac enhancers with diverse functional architectures. PLoS Genet. (2013) 9:e1003195. doi: 10.1371/journal.pgen.1003195

41. Han Z, Olson E. Hand is a direct target of Tinman and GATA factors during Drosophila cardiogenesis and hematopoiesis. Development. (2005) 132:3525-36. doi: 10.1242/dev.01899

42. Geier C, Perrot A, Ozcelik C, Binner P, Counsell D, Hoffmann K, et al. Mutations in the human muscle LIM protein gene in families with hypertrophic cardiomyopathy. Circulation. (2003) 107:1390-5. doi: 10.1161/01.CIR.0000056522.82563.5F

43. Hobert O, Westphal H. Functions of LIM-homeobox genes. Trends Genet. (2000) 16:75-83. doi: 10.1016/S0168-9525(99)01883-1

44. Mohapatra B, Jimenez S, Lin J, Bowles K, Coveler K, Marx J, et al. Mutations in the muscle LIM protein and alpha-actinin-2 genes in dilated cardiomyopathy and endocardial fibroelastosis. Mol Genet Metab. (2003) 80:207-15. doi: 10.1016/S1096-7192(03)00142-2

45. Sagave JF, Moser M, Ehler E, Weiskirchen S, Stoll D, Günther K, et al. Targeted disruption of the mouse Csrp2 gene encoding the cysteineand glycine-rich LIM domain protein CRP2 result in subtle alteration of cardiac ultrastructure. BMC Developmental Biology. (2008) 8:80. doi: 10.1186/1471-213X-8-80

46. Mery A, Taghli-Lamallem O, Clark KA, Beckerle MC, Wu X, Ocorr K, et al. The Drosophila muscle LIM protein, Mlp84B, is essential for cardiac function. J Exp Biol. (2008) 211:15-23. doi: 10.1242/jeb.012435

47. Clark K, Kadrmas J. Drosophila melanogaster muscle LIM protein and alphaactinin function together to stabilize muscle cytoarchitecture: a potential role for Mlp84B in actin-crosslinking. Cytoskeleton. (2013) 70:304-16. doi: $10.1002 / \mathrm{cm} .21106$

48. Stronach BE, Renfranz PJ, Lilly B, Beckerle MC. Muscle LIM proteins are associated with muscle sarcomeres and require dMEF2 for their expression during Drosophila myogenesis. Mol Biol Cell. (1999) 10:2329-42. doi: $10.1091 / \mathrm{mbc}$.10.7.2329

49. Johnson E, Sherry T, Ringo J, Dowse H. Modulation of the cardiac pacemaker of Drosophila: cellular mechanisms. J Comp Physiol B. (2002) 172:227-36. doi: 10.1007/s00360-001-0246-8

50. Folkers E, Drain P, Quinn W. Radish, a Drosophila mutant deficient in consolidated memory. Proc Natl Acad Sci USA. (1993) 90:8123-7. doi: $10.1073 /$ pnas.90.17.8123

51. Clark K, Bland J, Beckerle M. The Drosophila muscle LIM protein, Mlp84B, cooperates with D-titin to maintain muscle structural integrity. J Cell Sci. (2007) 120:2066-77. doi: 10.1242/jcs.000695

52. Waltzer L, Gobert V, Osman D, Haenlin M. Transcription factor interplay during Drosophila haematopoiesis. Int J Dev Biol. (2010) 54:1107-15. doi: 10.1387/ijdb.093054lw

53. Vlisidou I, Wood W. Drosophila blood cells and their role in immune responses. FEBS J. (2015) 282:1368-82. doi: 10.1111/febs.13235

54. Wu C, Yuan M, Gao Y, Sun W. LMO2 functional and transcriptional regulatory profiles in hematopoietic cells. Leuk Res. (2018) 75:11-4. doi: 10.1016/j.leukres.2018.10.013

55. Valge-Archer V, Forster A, Rabbitts TH. The LMO1 and LDB1 proteins interact in human $\mathrm{T}$ cell acute leukaemia with the chromosomal translocation $\mathrm{t}(11 ; 14)(\mathrm{p} 15 ; \mathrm{q} 11)$. Oncogene. (1998) 17:3199-202. doi: $10.1038 /$ sj.onc. 1202353

56. Osada H, Grutz G, Axelson H, Forster A, Rabbitts TH. Association of erythroid transcription factors: complexes involving the LIM protein RBTN2 and the zinc-finger protein GATA1. Proc Natl Acad Sci USA. (1995) 92:9585-9. doi: 10.1073/pnas.92.21.9585

57. Muratoglu S, Hough B, Mon S, Fossett N. The GATA factor serpent crossregulates lozenge and u-shaped expression during Drosophila blood cell development. Dev Biol. (2007) 311:636-49. doi: 10.1016/j.ydbio.2007.08.015

58. Minakhina S, Tan W, Steward R. JAK/STAT and the GATA factor Pannier control hemocyte maturation and differentiation in Drosophila. Dev Biol. (2011) 352:308-16. doi: 10.1016/j.ydbio.2011.01.035

59. Haenlin M, Cubadda Y, Blondeau F, Heitzler P, Lutz Y, Simpson P, et al. Transcriptional activity of pannier is regulated negatively by 
heterodimerization of the GATA DNA binding domain with a cofactor encoded by the u-shaped gene of Drosophila. Genes Dev. (1997) 11:3096-108. doi: 10.1101/gad.11.22.3096

Conflict of Interest: The authors declare that the research was conducted in the absence of any commercial or financial relationships that could be construed as a potential conflict of interest.
Copyright (ㄷ 2021 She, Tang, Jiang and Zeng. This is an open-access article distributed under the terms of the Creative Commons Attribution License (CC BY). The use, distribution or reproduction in other forums is permitted, provided the original author(s) and the copyright owner(s) are credited and that the original publication in this journal is cited, in accordance with accepted academic practice. No use, distribution or reproduction is permitted which does not comply with these terms. 